\title{
Localizations of tensor products
}

\author{
Manfred Dugas $(*)$ - Kelly Aceves $(*)$ - Bradley Wagner $(*)$
}

ABstract - A homomorphism $\lambda: A \rightarrow B$ between $R$-modules is called a localization if for all $\varphi \in \operatorname{Hom}_{R}(A, B)$ there is a unique $\psi \in \operatorname{Hom}_{R}(B, B)$ such that $\varphi=\psi \circ \lambda$. We investigate localizations of tensor products of torsion-free abelian groups. For example, we show that the natural multiplication map $\mu: R \otimes R \rightarrow R$ is a localization if and only if $R$ is an E-ring.

KEYWORDS. Torsion-free abelian groups, tensor products, localizations.

Mathematics Subject Classification (1991). Primary 20K30, 15A15; Secondary $46 \mathrm{M} 05$.

\section{Introduction}

Let $R$ denote some ring. Many notions in the theory of $R$-modules can be stated in terms of some universal property. Here are some examples:

(1) The $R$-module $F$ is free with basis $B$ if $B$ is a subset of $F$ such that for any $R$-module $X$ and any function $f: B \rightarrow X$ there exists a unique homomorphism $\varphi: F \rightarrow X$ such that $\left.\varphi\right|_{B}=f$.

(2) The $R$-module $G$ is small, if for each family $\left\{X_{i}: i \in I\right\}$ of $R$-modules the abelian group $\operatorname{Hom}_{R}\left(G, \bigoplus_{i \in I} X_{i}\right)$ is naturally isomorphic to $\bigoplus_{i \in I} H_{R} m_{R}\left(G, X_{i}\right)$.

(3) The $R$-module $G$ is strongly slender [9] if for each family $\left\{X_{i}: i \in I\right\}$ of $R$-modules the abelian group $\operatorname{Hom}_{R}\left(\prod_{i \in I} X_{i}, G\right)$ is naturally isomorphic to $\bigoplus_{i \in I} \operatorname{Hom}_{R}\left(X_{i}, G\right)$.

(*) Indirizzo degli A.: Department of Mathematics, Baylor University, Waco, Texas 76798, USA.

E-mail: manfred_dugas@baylor.edu kelly_fouts@baylor.edu bradley_wagner@baylor.edu 
(4) Let $\lambda \in \operatorname{Hom}_{R}(A, B)$ be a homomorphism. We call $\lambda$ a split homomorphism if for any $R$-module $X$ and any $\varphi \in \operatorname{Hom}_{R}(A, X)$ there exists some $\psi \in \operatorname{Hom}_{R}(B, X)$ such that $\varphi=\psi \circ \lambda$. If one considers the case of $X=A$ and $\varphi=i d_{A}$, then it is easy to see that $\lambda$ is indeed a splitting homomorphism, i.e. $\lambda$ is injective and $\lambda(A)$ is a direct summand of $B$.

(5) The $R$-module $G$ is injective if for any $R$-module $X$ and any submodule $K$ of $X$ and any $\varphi \in \operatorname{Hom}_{R}(K, G)$ there exists some $\psi \in$ $\operatorname{Hom}_{R}(X, G)$ such that $\varphi=\left.\psi\right|_{K}$. Note that "projective" is the dual of "injective".

(6) Tensor products: To simplify notation, let us assume $R=Z$. Let $A, B$ and $T$ be abelian groups and $\tau: A \times B \rightarrow T$ a bilinear map. The pair $(T, \tau)$ is a tensor product of $A$ and $B$, if for any abelian group $X$ and any bilinear map $\sigma: A \times B \rightarrow X$, there exists a unique $\psi \in \operatorname{Hom}(T, X)$ such that $\sigma=\psi \circ \tau$. It is well known, of course, that tensor products exist and are unique up to isomorphism.

It would be easy to continue this list. All these definitions can be modified by restricting the $X$ 's. Let us do this. We get

$\left(1^{s}\right)$ In (1), replace " $X$ " by " $F$ ". A module $F$ satisfying $\left(1^{s}\right)$ is called selffree with basis $B$, c.f. [5].

$\left(2^{s}\right)$ In (2), replace all the " $X_{i}$ " by " $G$ ". Such a module $G$ is called selfsmall. This notion has been studied by many authors, c.f. [3] and the literature referenced there.

$\left(3^{s}\right)$ In (3), replace all the " $X_{i}$ " by " $G$ ". Such a module is called strongly self-slender, c.f. [9].

$\left(4^{s}\right)$ In (4) replace " $X$ " by " $B$ " and add the condition that the map $\psi$ is unique. Such a homomorphism $\lambda: A \rightarrow B$ is called a localization of $A$. There is a large amount of literature on localizations. See for instance [6] and [7] and the papers referenced there.

$\left(5^{s}\right)$ In (5), replace " $X$ " by " $G$ ". Then $G$ is called quasi-injective. If $R=Z$ and $K$ is restricted to $p$-pure subgroups of $G$, then $G$ is called quasi$p$-pure-injective, or qppi for short. Again, there is a lot of literature on this topic dating back to the 1970 's, c.f. [1] or [13].

In this paper we focus on a specification $\left(6^{s}\right)$ of $(6)$ : 
Definition 1. Let $R$ be a ring, $A=A_{R}$ a right $R$-module, $B={ }_{R} B$ a left $R$-module and $T$ some Abelian group. Following [12, page 207], we call a map $\tau: A \times B \rightarrow T$ a middle linear map if $\tau$ is bilinear and $\tau(a r, b)=\tau(a, r b)$ for all $a \in A, b \in B$ and $r \in R$. Let $\operatorname{Midlin}_{R}(A, B ; T) d e-$ note the set of all middle linear maps from $A \times B$ into $T$. We call the pair $(T, \tau)$ a qutensor product of $A, B$ over $R$ if for all $\sigma \in \operatorname{Midlin}_{R}(A, B ; T)$, there exists a unique homomorphism $\psi \in \operatorname{Hom}_{\mathrm{Z}}(T, T)$ such that $\sigma=\psi \circ \tau$. The $\operatorname{map} \otimes: A \times B \rightarrow A \otimes_{R} B$ is a middle linear map with $\otimes(a, b)=a \otimes b$ for all $a \in A, b \in B$.

It follows from the definitions that qutensor products are a combination of the notions in $\left(4^{s}\right)$ and (6):

The pair $(T, \tau)$ is a qutensor product of the modules $A, B$ if and only if there exists a localization $\lambda: A \otimes_{R} B \rightarrow T$ such that $\tau=\lambda \circ \otimes$, where $\otimes: A \times B \rightarrow A \otimes_{R} B$ is the natural map with $\otimes(a, b)=a \otimes b$ for all $a \in A$ and $b \in B$.

In this paper we concern ourselves with localizations of tensor products of torsion-free abelian groups $A, B$.

After some preliminaries in Section 2, we study localizations of arbitrary direct sums of modules in Section 3. In Section 4, we look at surjective localizations and find conditions for $p$-reduced torsion-free abelian groups having a $p$-reduced tensor product. In Section 5, we use the absolute E-rings constructed in [10] and [11] to obtain absolute localizations of torsion-free abelian groups that are $p$-reduced for infinitely many primes $p$. In Section 6 , we present some examples of some surprising properties of tensor products of reduced torsion-free abelian groups. For example, there exists a strongly indecomposable reduced abelian group $G$ such that $G \otimes G$ is reduced and completely decomposable. In Section 7, we consider torsion-free abelian groups of finite rank whose $p$-rank is less than their rank. We find an example of such a group $G$ of rank 4 and $p$-rank 2 such that $G \otimes G$ is not reduced, but $G$ has no pure subgroups of rank at least 2 but of $p$-rank 1 . (It is well known that $G \otimes G$ is not $p$-reduced if $G$ has rank at least 2 and $p$ rank 1.) We also find a quasi-isomorphism invariant for such groups. In the last section, we take a glimpse at zero product determinated algebras, c.f. [4]. Let $1 \in R$ be a ring. Then there exists an epimorphism $\mu: R \otimes_{\mathrm{Z}} R \rightarrow R$ with $\mu(a \otimes b)=a b$ for all $a, b \in R$. We show that $\mu$ is a localization if and only if $R$ is an E-ring. 


\section{Definitions and First Results}

Recall the following, well established

Definition 2. Let $M, L, X$ be modules over some ring and $\lambda \in$ $\operatorname{Hom}(M, L)$. Then $\lambda \perp X$ provided that for any $\alpha \in \operatorname{Hom}(M, X)$ there $i$ s a unique $\beta \in \operatorname{Hom}(L, X)$ such that $\alpha=\beta \circ \lambda$. If $\lambda \perp L$, then $\lambda$ is called a localization of $M$. Abusing notations, sometimes $L$ is called a localization of $M$.

Next we show that qutensors are exactly the localizations of the ordinary tensor product of the modules.

Proposition 1. Let $A_{R}$ and ${ }_{R} B$ be modules and $\tau \in \operatorname{Midlin}_{R}(A, B ; D)$. Then $\tau$ factors through $\otimes$, i.e. there exists $\lambda: A \otimes_{R} B \rightarrow D$ such that $\tau=\lambda \circ \otimes$. Moreover, $(D, \tau)$ is a qutensor product of $A, B$ if and only if $\lambda: A \otimes_{R} B \rightarrow D$ is a localization of $A \otimes_{R} B$.

Proof. By the universal property of the tensor product, there is a unique $\lambda \in \operatorname{Hom}\left(A \otimes_{R} B, D\right)$ such that $\tau=\lambda \circ \otimes$. Assume that $\lambda$ is a localization and let $\sigma: A \times B \rightarrow D$ be a bilinear map. By the universal property of the tensor product, there exists a unique map $\delta \in \operatorname{Hom}\left(A \otimes_{R} B, D\right)$ such that $\sigma=\delta \circ \otimes$. Then there is a unique $\beta \in \operatorname{End}(D)$ such that $\delta=\beta \circ \lambda$ and we have that $\sigma=\beta \circ \lambda \circ \otimes=\beta \circ \tau$. On the other hand, assume that $\sigma=\gamma \circ \tau$. We infer that $\gamma \circ \tau=\gamma \circ \lambda \circ \otimes=\beta \circ \lambda \circ \otimes$ and thus $\gamma \circ \lambda=\beta \circ \lambda$. We conclude that $\gamma=\beta$ and we have that $(D, \tau)$ is a qutensor of $A, B$.

For the other direction, let $\alpha \in \operatorname{Hom}\left(A \otimes_{R} B, D\right)$ and put $\sigma=\alpha \circ \otimes$, a middle linear map from $A \times B$ into $D$. Thus there exists a unique $\beta \in \operatorname{End}(D)$ such that $\sigma=\beta \circ \tau$ and thus $\alpha \circ \otimes=\beta \circ \lambda \circ \otimes$, which implies that $\alpha=\beta \circ \lambda$. If $\beta^{\prime} \in \operatorname{End}(D)$ is some other map with $\alpha=\beta^{\prime} \circ \lambda$, then $\sigma=\alpha \circ \otimes=\beta^{\prime} \circ \lambda \circ \otimes=\beta \circ \lambda \circ \otimes$ and we infer $\beta \circ \tau=\beta^{\prime} \circ \tau$ and thus $\beta=\beta^{\prime}$. This shows that $\lambda$ is a localization.

\section{Localizations of Direct Sums of Modules}

We begin with:

Proposition 2. Let $\lambda: A=\bigoplus_{i \in I} A_{i} \rightarrow D$ be a localization, i.e. $\lambda \perp D$. Then there exists a set $\left\{\gamma_{i}: i \in I\right\}$ of orthogonal idempotents in $\operatorname{End}(D)$ such that: 


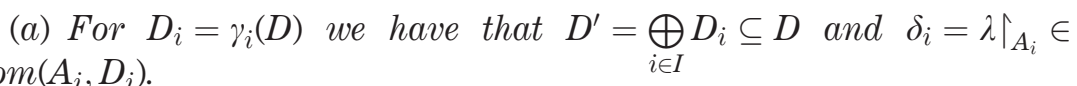
$\operatorname{Hom}\left(A_{i}, D_{i}\right)$.

(b) $\delta_{j} \perp D_{i}$ for all $i, j \in I$.

(c) $\operatorname{ker}(\lambda)=\bigoplus_{i \in I}\left(A_{i} \cap \operatorname{ker}(\lambda)\right)$.

(d) If the index set I is finite, then $D=D^{\prime}$.

Proof. Define $\lambda_{i}: A \rightarrow D$ by $\left.\lambda_{i}\right|_{A_{i}}=\lambda \uparrow_{A_{i}}$ and $\lambda_{i}\left(A_{j}\right)=\{0\}$ for all $i \neq j \in I$. Then there exist unique $\gamma_{i} \in \operatorname{End}(D)$ such that

$\lambda_{i}=\gamma_{i} \circ \lambda$ for all $i \in I$.

Let $a=\sum_{j \in I} a_{j} \in A$ where $a_{j} \in A_{j}$. Then $\left(\gamma_{i} \circ \lambda_{i}\right)(a)=\left(\gamma_{i} \circ \lambda_{i}\right)\left(a_{i}\right)=$ $\left(\gamma_{i} \circ \lambda\right)\left(a_{i}\right)=\lambda_{i}\left(a_{i}\right)=\lambda_{i}(a)$ and we have:

$\lambda_{i}=\gamma_{i} \circ \lambda_{i}$ for all $i \in I$.

Now we have $\left(\gamma_{i}^{2}\right) \circ \lambda=\gamma_{i} \circ\left(\gamma_{i} \circ \lambda\right)=\gamma_{i} \circ \lambda_{i}=\lambda_{i}=\gamma_{i} \circ \lambda$ and we infer that $\gamma_{i}$ is an idempotent element of $\operatorname{End}(D)$.

Let $i \neq j \in I$ and $a \in A$ as above. Then $\left(\gamma_{i} \circ \gamma_{j} \circ \lambda\right)(a)=\left(\gamma_{i} \circ \lambda_{j}\right)(a)=$ $\gamma_{i}\left(\lambda_{j}\left(a_{j}\right)\right)=\gamma_{i}\left(\lambda\left(a_{j}\right)\right)=\lambda_{i}\left(a_{j}\right)=0$. This shows that $\left(\gamma_{i} \circ \gamma_{j}\right) \circ \lambda=0=0 \circ \lambda$ and it follows that $\gamma_{i} \circ \gamma_{j}=0$ for all $i \neq j \in I$. We infer that $\left\{\gamma_{i}: i \in I\right\}$ is a set of orthogonal idempotents and thus $D^{\prime}=\bigoplus_{i \in I} D_{i}$ is a submodule of $D$ where $D_{i}=\gamma_{i}(D)$.

Let $a_{i} \in A_{i}$. Then $\lambda\left(a_{i}\right)=\lambda_{i}\left(a_{i}\right)=\gamma_{i}\left(\lambda_{i}\left(a_{i}\right)\right) \subseteq \gamma_{i}(D)=D_{i}$. This shows that $\delta_{i} \in \operatorname{Hom}\left(A_{i}, D_{i}\right)$.

Note that $D=D_{i} \oplus \operatorname{ker}\left(\gamma_{i}\right)$ and $\gamma_{j}(D) \subseteq \operatorname{ker}\left(\gamma_{i}\right)$ for all $i \neq j \in I$.

To show that $\delta_{i}=\lambda \Gamma_{A_{i}}: A_{i} \rightarrow D_{i}$ is orthogonal to $D_{j}$, let $\eta \in \operatorname{Hom}\left(A_{i}, D_{j}\right)$ for some $j \in I$. Then $\eta$ naturally extends to a map $\eta^{\prime}$ from $A$ to $D$ by setting the map $\eta^{\prime}$ equal to 0 on the other summands of $A$. Then there exists a unique $\operatorname{map} \theta: D \rightarrow D$ such that $\eta^{\prime}=\theta \circ \lambda$. Let $a=a_{i}+a^{(i)} \in A$ where $a_{i} \in A_{i}$ and $a^{(i)} \in \bigoplus_{i \neq j \in I} A_{j}$. Then $(\theta \circ \lambda)(a)=(\theta \circ \lambda)\left(a_{i}\right)+(\theta \circ \lambda)\left(a^{(i)}\right)=\eta^{\prime}(\alpha)=\eta\left(a_{i}\right) \in D_{j}$. It follows that $(\theta \circ \lambda)\left(a^{(i)}\right)=0$ by setting $a_{i}=0$. Since $\gamma_{j}$ acts as the identity map on $D_{j}$, we get $\left(\gamma_{j} \circ \theta \uparrow_{D_{i}}\right) \circ\left(\lambda \uparrow_{A_{i}}\right)=\eta$. Let $\theta_{i}=\gamma_{j} \circ \theta \uparrow_{D_{i}} \in \operatorname{Hom}\left(D_{i}, D_{j}\right)$. Then $\eta=\theta_{i} \circ \delta_{i}$.

Next we need to show that the map $\theta_{i}$ is unique with that property. Let $\theta_{i}^{\prime}$ be another such map, and note that $\lambda\left(A^{(i)}\right) \subseteq \operatorname{ker}\left(\gamma_{i}\right)$. Let $\theta^{\prime} \in \operatorname{End}(D)$ with $\theta^{\prime} \uparrow_{D_{i}}=\theta_{i}^{\prime}$ and $\theta^{\prime}\left(\operatorname{ker}\left(\gamma_{i}\right)\right)=\{0\}$. It follows that $\eta^{\prime}=\theta^{\prime} \circ \lambda$ and since $\lambda \perp D$, we infer that $\theta=\theta^{\prime}$ and thus $\theta_{i}=\theta_{i}^{\prime}$.

Let $a=\sum_{i} a_{i} \in \operatorname{ker}(\lambda)$ where $a_{i} \in A_{i}$. Then $0=\sum_{i} \lambda\left(a_{i}\right)=\sum_{i} \lambda_{i}\left(a_{i}\right)=$ $\sum_{i} \gamma_{i}\left(\lambda_{i}\left(a_{i}\right)\right) \in \bigoplus_{i \in I} D_{i}$ and it follows that $\lambda\left(a_{i}\right)=0$ for all $i \in I$. This shows that $\operatorname{ker}(\lambda) \subseteq \bigoplus_{i \in I}\left(A_{i} \cap \operatorname{ker}(\lambda)\right) \subseteq \operatorname{ker}(\lambda)$ and (c) follows. 
Now assume that $I$ is finite. Then $i d_{D} \circ \lambda=\sum_{i \in I} \lambda_{i}=\sum_{i \in I} \gamma_{i} \circ \lambda=\left(\sum_{i \in I} \gamma_{i}\right) \circ \lambda$ and it follows that $i d_{D}=\sum_{i \in I} \gamma_{i}$.

Corollary 1. Given abelian groups $D$ and $A=\bigoplus_{i \in I} A_{i}$ with I finite. Let $\lambda \in \operatorname{Hom}(A, D)$. If $\lambda \perp D$, then $D=\bigoplus_{i \in I} D_{i}$ and $\delta_{i}=\lambda \uparrow_{A_{i}} \in \operatorname{Hom}\left(A_{i}, D_{i}\right)$ satisfies $\delta_{i} \perp D_{j}$ for all $i, j \in I$.

Conversely, if $D=\bigoplus_{i \in I} D_{i}$ and $\lambda_{i} \in \operatorname{Hom}\left(A_{i}, D_{i}\right)$ with $\lambda_{i} \perp D_{j}$ for all $i, j \in I$, then $\lambda=\bigoplus_{i \in I} \lambda_{i} \perp \stackrel{i \in I}{\perp}$.

COROLlary 2. Let $A=\bigoplus_{i \in I} A_{i}$ with finite index set $I$ and $K$ a subgroup of $A$. The canonical map $\pi: A \rightarrow A / K$ is a localization if and only if

(1) The canonical maps $\pi_{i}: A_{i} \rightarrow A_{i} /\left(K \cap A_{i}\right)$ are localizations for all $i \in I$ and

(2) $K=\bigoplus_{i \in I}\left(K \cap A_{i}\right)$ and $\pi_{i} \perp\left(A_{j} /\left(K \cap A_{j}\right)\right)$ for all $i, j \in I$.

Proof. It follows from Proposition 2 that (1) and (2) are necessary. Suppose (1) and (2) hold. Let $\varphi \in \operatorname{Hom}(A, A / K)$ and note that $A / K \equiv$ $\bigoplus_{i \in I}\left(A_{i} /\left(A_{i} \cap K\right)\right)$ because of (2). There exist $\varphi_{j i}: A_{i} \rightarrow A_{j} /\left(A_{i} \cap K\right)$ such that $\varphi$ is represented by the matrix $\left[\varphi_{j i}\right]$. For each $\varphi_{j i}$ there is a unique $\psi_{j i}: A_{i} /\left(A_{i} \cap K\right) \rightarrow A_{j} /\left(A_{j} \cap K\right)$ such that $\varphi_{j i}=\psi_{j i} \circ \pi_{i}$. It follows from the definitions that $\psi=\left[\psi_{j i}\right] \in \operatorname{End}(A / K)$ is the desired map.

Corollary 3. With the above notation, if $A_{i} \cong A_{j}$ then $D_{i} \cong D_{j}$ for any $i, j \in I$.

Proof. Let $\sigma_{j i}: A_{i} \rightarrow A_{j}$ be an isomorphism with $\sigma_{i j}: A_{j} \rightarrow A_{i}$ the inverse map. Extend $\sigma_{j i}$ to $\sigma_{j i}^{\prime}: A \rightarrow A$ by setting $\sigma_{j i}^{\prime}\left(A_{\alpha}\right)=\{0\}$ for all $i \neq \alpha \in I$. Then $\lambda \circ \sigma_{j i}^{\prime} \in \operatorname{Hom}(A, D)$ and there exists a unique $\delta_{j i} \in \operatorname{End}(D)$ such that

(1) $\lambda \circ \sigma_{j i}^{\prime}=\delta_{j i} \circ \lambda$. In a similar fashion we get

(2) $\lambda \circ \sigma_{i j^{\prime}}^{\prime}=\delta_{i j} \circ \lambda$.

It follows that $\gamma_{j} \circ \lambda=\lambda_{j}=\left(\lambda \circ \sigma_{j i}^{\prime}\right) \circ \sigma_{i j}^{\prime}=\left(\delta_{j i} \circ \lambda\right) \circ \sigma_{i j}^{\prime}=\delta_{j i} \circ\left(\lambda \circ \sigma_{i j}^{\prime}\right)=$ $\left(\delta_{j i} \circ \delta_{i j}\right) \circ \lambda$. We infer

(3) $\gamma_{j}=\delta_{j i} \circ \delta_{i j}$ as well as $\gamma_{i}=\delta_{i j} \circ \delta_{j i}$. It follows that

(4) $\gamma_{i} \circ \delta_{i j}=\delta_{i j} \circ \delta_{j i} \circ \delta_{i j}=\delta_{i j} \circ \gamma_{j}$ and $\gamma_{j} \circ \delta_{j i}=\delta_{j i} \circ \delta_{i j} \circ \delta_{j i}=\delta_{j i} \circ \gamma_{i}$. 
Taking the restrictions to $D_{j}, D_{i}$ we get

(5) $\left.\delta_{i j}\right|_{D_{j}}=\left.\gamma_{i} \circ \delta_{i j}\right|_{D_{j}}: D_{j} \rightarrow D_{i}$ as well as $\left.\delta_{j i}\right|_{D_{i}}=\gamma_{j} \circ \delta_{j i} \hat{1}_{D i}: D_{i} \rightarrow D_{j}$.

The equations (3) now imply that $\left.\delta_{i j}\right|_{D_{j}}$ and $\left.\delta_{j i}\right|_{D_{i}}$ are a pair of inverse isomorphisms and we have $D_{i} \cong D_{j}$.

Proposition 3. Same notations as above. If $D$ is slender, then $D=\bigoplus_{i \in I} D_{i}$.

Proof. Since $\lambda$ is a localization, we have that $\prod_{i \in I} \operatorname{Hom}\left(A_{i}, D\right) \cong$ $\operatorname{Hom}\left(\bigoplus_{i \in I} A_{i}, D\right)=\operatorname{Hom}(A, D) \cong \operatorname{End}(D)$. The latter isomorphism * $\operatorname{End}(D) \rightarrow$ $\operatorname{Hom}(A, D)$ is given by $\varphi^{*}=\varphi \circ \lambda$ for all $\varphi \in \operatorname{End}(D)$. Let \# denote the inverse of *.

Pick an element $d \in D$.

Define a map $\sigma_{d}: \operatorname{End}(D) \rightarrow D$ by $\sigma_{d}(\varphi)=\varphi(d)$ for all $\varphi \in \operatorname{End}(D)$. This gives rise to a homomorphism $\tau_{d}: \prod_{i \in I} \operatorname{Hom}\left(A_{i}, D\right) \rightarrow D$. Since $D$ is slender, there exists a cofinite subset $J$ of $I$ such that $\tau_{d}\left(\prod_{i \in J} \operatorname{Hom}\left(A_{i}, D\right)\right)=\{0\}$. Note that $\left(\lambda_{i}\right)^{\#}=\gamma_{i}$ for all $i \in I$ and $\tau_{d}\left(\lambda_{j}\right)=0$ for all $j \in J$. But now we have $0=\tau_{d}\left(\lambda_{j}\right)=\gamma_{j}(d)$ for all $j \in J$. We infer that $\sum_{i \in I} \gamma_{i}(d)$ is a finite sum for all $d \in D$ and thus $\gamma=\sum_{i \in I} \gamma_{i} \in \operatorname{End}(D)$ with $\gamma \circ \lambda=\lambda=i d_{D} \circ \lambda$. It follows that $\gamma=i d_{D}$ and thus $D=\bigoplus_{i \in I} D_{i}$.

DEFINITION 3. Let $(A, D)$ be a pair of abelian groups. We call this pair relatively semi-rigid, if each $0 \neq \varphi \in \operatorname{Hom}(A, D)$ is injective.

For example, if $A \cong Z$ and $D$ is torsion-free, then the pair $(A, D)$ is relatively semi-rigid.

Proposition 4. Let I be an index set and $A_{i}, D_{i}$ be abelian groups for all $i \in I$. Moreover, let $\lambda_{i} \in \operatorname{Hom}\left(A_{i}, D_{i}\right)$ for all $i \in I$ such that:

(1) $\lambda_{i} \perp D_{j}$ for all $i, j \in I$.

(2) For all $i \in I$, we have that the pair $\left(A_{i}, D_{j}\right)$ is relatively semi-rigid for all but finitely many $j \in I$.

Then $\lambda=\bigoplus_{i \in I} \lambda_{i}: A=\bigoplus_{i \in I} A_{i} \rightarrow D=\bigoplus_{i \in I} D_{i}$ is a localization, i.e. $\lambda \perp D$. 
Proof. Let $\varphi \in \operatorname{Hom}(A, D)$. Then $\varphi=\left[\varphi_{j i}\right]$ where $\varphi_{j i} \in \operatorname{Hom}\left(A_{i}, D_{j}\right)$ and for all $i \in I$ and $a_{i} \in A_{i}$ we have $\varphi_{j i}\left(a_{i}\right)=0$ for all but finitely many $j \in I$. By (2) we infer that $\varphi_{j i}=0$ for all but finitely many $j \in I$. By (1), there exist unique $\gamma_{j i} \in \operatorname{Hom}\left(D_{i}, D_{j}\right)$ such that $\varphi_{j i}=\gamma_{j i} \circ \lambda_{i}$. Note that $\gamma_{j i}=0$ whenever $\varphi_{j i}=0$. This implies that $\gamma=\left[\gamma_{j i}\right] \in \operatorname{End}(D)$ and $\varphi=\gamma \circ \lambda$. The uniqueness of $\gamma$ with that property follows immediately.

Corollary 4. Let I be an index set and $A_{i}, D_{i}$ be abelian groups for all $i \in I$. Moreover, let $\lambda_{i} \in \operatorname{Hom}\left(A_{i}, D_{i}\right)$ for all $i \in I$ such that:

(1) $\lambda_{i} \perp D_{j}$ for all $i, j \in I$.

(2) For all $i \in I$, we have that $\operatorname{Hom}\left(A_{i}, D_{j}\right)=0$ for all but finitely many $j \in I$.

Then $\lambda=\bigoplus_{i \in I} \lambda_{i}: A=\bigoplus_{i \in I} A_{i} \rightarrow D=\bigoplus_{i \in I} D_{i}$ is a localization, i.e. $\lambda \perp D$.

\section{Surjective Localization}

Proposition 5. Let B be a subgroup of the abelian group $A$ such that:

(1) B is fully invariant in $A$.

(2) The natural map $\operatorname{End}(A) \rightarrow \operatorname{Hom}(A, A / B)$ is surjective.

Then the canonical map $\pi: A \rightarrow A / B$ is a localization.

Proof. Let $\varphi: A \rightarrow A / B$ be a homomorphism. By (2), there is some $\theta \in \operatorname{End}(A)$ such that $\varphi=\pi \circ \theta$. Define $\psi: A / B \rightarrow A / B$ by $\psi(a+B)=$ $\theta(a)+B$. Note that $\psi$ is well defined by (1) and it follows that $\varphi=\psi \circ \pi$. Since $\pi$ is surjective, we infer that $\psi$ is unique with that property.

Let $G$ be a torsion-free abelian group. For $a \in G$, let $\|a\|$ denote the type of $a \in G$ and $G(\tau)=\{g \in G:\|g\| \geq \tau\}$. A type $\tau=\left(\tau_{p}\right)_{p \in \mathbb{P}}$ is called idempotent (or ring) type, if $\tau_{p} \in\{0, \infty\}$ for all primes $p \in \mathbb{P}$. Moreover $\|G\|$ denotes the set of all types of the elements of $G$.

Proposition 6. Let $G$ be a torsion-free abelian group. Suppose the type $\tau$ is an element in $\|A\|$ and of ring type, i.e. for $\tau=\left(\tau_{p}\right)_{p \in \mathbb{P}}$ we have $\tau_{p} \in\{0, \infty\}$. Then $(G / G(\tau))(\tau)=\{0\}$ and for all $\varphi \in \operatorname{Hom}(G, G / G(\tau))$ we have $G(\tau) \subseteq \operatorname{ker}(\varphi)$. Moreover, the natural map $\pi: G \rightarrow G / G(\tau)$ is a localization. 
Proof. Let $x \in G$ such that $\|x+G(\tau)\| \geq \tau$. Let $p$ be a prime such that $\tau_{p}=\infty$. We have that for all $n \in \mathbb{N}$, there is some $x_{n} \in G$ such that $p^{n} x_{n}-x=k_{n} \in G(\tau)$. Now $k_{n}$ is $p$-divisible in $G$ and it follows that $x$ is $p$ divisible in $G$. This implies that $x \in G(\tau)$ and thus $x+G(\tau)=0$. Note that $\varphi(G(\tau)) \subseteq(G / G(\tau))(\tau)=\{0\}$. Thus $\varphi$ induces a map $\tilde{\varphi} \in \operatorname{End}(G / G(\tau))$ by $\widetilde{\varphi}(x+G(\tau))=\varphi(x)$. It follows that $\varphi=\widetilde{\varphi} \circ \pi$ and $\widetilde{\varphi}$ is unique with this property because $\pi$ is surjective. This shows that $\pi$ is a localization.

Let $p$ be a prime integer. Then $Z_{p}=\left\{\frac{z}{n}: z \in Z, n \in \mathbb{N}, \operatorname{gcd}(p, n)=1\right\}$ denotes the ring of integers localized at $p$. The torsion-free abelian group is called $p$-locally free if $G \otimes_{Z} Z_{p}$ is a free $Z_{p}$-module.

REMARK 1. Let $A^{\prime}, B^{\prime}$ be pure subgroups of the torsion-free abelian groups $A, B$ respectively. Then $A^{\prime} \otimes B^{\prime}$ is a pure subgroup of $A \otimes B$.

To see this, note that $0 \rightarrow A^{\prime} \otimes B \rightarrow A \otimes B \rightarrow\left(A / A^{\prime}\right) \otimes B \rightarrow 0$ is pureexact, and $0 \rightarrow A^{\prime} \otimes B^{\prime} \rightarrow A^{\prime} \otimes B \rightarrow A^{\prime} \otimes\left(B / B^{\prime}\right) \rightarrow 0$ is pure-exact and purity is transitive. This shows:

Proposition 7. Let $A, B$ be torsion-free abelian groups. Then $A \otimes B$ is $p$-reduced if and only if $A^{\prime} \otimes B^{\prime}$ is p-reduced for all pure, finite rank subgroups $A^{\prime}, B^{\prime}$ of $A, B$ respectively.

THeOREm 1. Let $A, B$ be torsion-free, p-reduced abelian groups such that all pure, finite rank subgroups of $A$ are $p$-locally free. Then $A \otimes B$ is p-reduced.

Proof. If there exists a non-zero element $w$ in $A \otimes B$ of infinite $p$ height, then there exists a pure, finite subgroup $A^{\prime}$ of $A$ such that $w$ is an element of $A^{\prime} \otimes B$. Now consider the localization $\left(A^{\prime} \otimes B\right)_{p}$ of $A^{\prime} \otimes B$ at the prime $p$. We have $\left(A^{\prime} \otimes B\right)_{p} \cong A_{p}^{\prime} \otimes B_{p} \cong \sum_{k} B_{p}$ as $Z_{p}$-modules since $A_{p}^{\prime}$ is a free $Z_{p}$-module of some rank $k$. Since $B$ is $p$-reduced, the $Z_{p}$-module $B_{p}$ has no elements of infinite $p$-height, which shows that no such element $w$ exists.

By an assertion of Warfield's, a torsion-free group $G$ of finite rank $m$ is $p$-locally free if and only if $m=r_{p}(G):=\operatorname{dim}_{Z / p Z}(G / p G)$. For the convenience of the reader, here is an outline of the proof: 
Let $\left\{a_{i}+p G: 1 \leq i \leq m\right\}$ be a basis of $G / p G$ and $B=\sum_{1 \leq i \leq m} a_{i} Z$. Then $\left\{a_{i}: 1 \leq i \leq m\right\}$ is $p$-independent and $B$ is a $p$-basic subgroup of $G$, i.e. $B$ is a free, $p$-pure subgroup of $G$ and $G / B$ is $p$-divisible and a torsion group with $(G / B)[p]=\{0\}$. It follows that $G \otimes Z_{p}=B \otimes Z_{p}$ is a free $Z_{p}$-module.

CoRollary 5. Let A, B be torsion-free, p-reduced abelian groups such that for all pure, finite rank subgroups $A^{\prime}$ of $A$ the rank of $A^{\prime}$ is equal to the p-rank $r_{p}\left(A^{\prime}\right)$ of $A^{\prime}$. Then $A \otimes B$ is p-reduced.

\section{Absolute Localizations}

Let $A$ be some algebraic structure that has some property $\mathcal{P}$. Then $A$ has property $\mathcal{P}$ absolutely if $A$ has property $\mathcal{P}$ in any generic extension of the set-theoretic universe in which $A$ was originally constructed. Let $\kappa(\omega)$ denote the first $\omega$-Erdös cardinal. In a remarkable paper [10], Göbel, Herden and Shelah constructed absolute E-rings $R$ of cardinality $\lambda$ for any infinite cardinal $\lambda<\kappa(\omega)$. Inspecting their proof, one realizes that the following result was shown:

THeorem 2. [10] Let $\lambda<\kappa(\omega)$ be a cardinal and Z[X] (resp. $\mathrm{Q}[X]$ ) the polynomial ring in $\lambda$ commuting variable over $Z$ (resp. Q). Then there exists a countable family $\left\{L_{i}: i<\omega\right\}$ of ideals of $Z[X]$ such that

(1) Each $L_{i}$ is a direct summand of the abelian group $Z[X]$ and

(2) $\left\{\varphi \in \operatorname{End}_{\mathrm{Q}}(\mathrm{Q}[X]): \varphi\left(\mathrm{Q} L_{i}\right) \subseteq \mathrm{Q} L_{i}\right.$ for all $\left.i<\omega\right\}=\mathrm{Q}[X] \cdot$ absolutely.

This version will appear in [11].

We will use this result to construct absolute localizations. First we show:

Lemma 1. Let $A$ be a commutative Q-algebra and $\mathcal{F}$ a family of ideals of A such that $\left\{\varphi \in\right.$ End $_{\mathrm{Q}}(A): \varphi(J) \subseteq J$ for all $\left.J \in \mathcal{F}\right\}=A$. Let $V$ be a Q-vector space. Then $\left\{\varphi \in H_{o m}\left(A, V \otimes_{\mathbb{Q}} A\right): \varphi(J) \subseteq V \otimes_{\mathbb{Q}} J\right.$ for all $\left.J \in \mathcal{F}\right\}=$ $H_{0 m}\left(A, V \otimes_{Q} A\right)$.

Proof. Let $B$ be a basis of the vector space $V$. Let $\pi_{b}: V \otimes_{\mathrm{Q}} A \rightarrow$ $b \otimes A$ be the natural projection with $\pi_{b}(c \otimes A)=\{0\}$ for all $b \neq c \in B$. Let $\varphi \in\left\{\varphi \in \operatorname{Hom}_{\mathrm{Q}}\left(A, V \otimes_{\mathrm{Q}} A\right): \varphi(J) \subseteq V \otimes_{\mathrm{Q}} J\right.$ for all $\left.J \in \mathcal{F}\right\}$. Then $\pi_{b} \circ \varphi: A \rightarrow b \otimes A \cong A$ and $\left(\pi_{b} \circ \varphi\right)(J) \subseteq \pi_{b}\left(V \otimes_{Q} J\right)=b \otimes J \cong J$. Thus, 
by hypothesis, $\left(\pi_{b} \circ \varphi\right)(x)=\left(\left(\pi_{b} \circ \varphi\right)(1)\right) x$ for all $x \in A$. It follows that $\varphi(x)=\sum_{b \in B} \pi_{b}(\varphi(x))=\sum_{b \in B}\left(\left(\pi_{b} \circ \varphi\right)(1)\right) x=\varphi(1) x$ and $\varphi$ is $A$-linear.

Let $G$ be a torsion-free abelian group and $P=\left\{p_{i}: i<\omega\right\}$ an infinite set of prime integers such that $G$ is $p$-reduced for all $p \in P$. Let $\tau_{i}$ denote the type of the subring $Z_{4}\left[\frac{1}{p_{i}}\right]$ of $Q$. With the notations of Theorem 2 , let $R=$ $\mathbb{Z}[X]+\sum_{i<\omega} Z_{[}\left[\frac{1}{p_{i}}\right] L_{i} \subseteq \mathbb{Q}[X]$. Then $R$ is the absolute E-ring constructed in [10]. Note that $R / Z[X]$ is a torsion abelian group. By (1) we have $Z[X]=$ $L_{i} \oplus T_{i}$ as abelian groups. Then $R / Z \mathbb{Z}[X]=\sum_{i<\omega}\left(\mathbb{Z}[X]+\mathbb{Z}\left[\frac{1}{p_{i}}\right] L_{i}\right) / \mathbb{Z}[X]=$ $\sum_{i<\omega}\left(T_{i} \oplus Z\left[\frac{1}{p_{i}}\right] L_{i}\right) /\left(T_{i} \oplus L_{i}\right) \cong \sum_{i<\omega}\left(Z\left[\frac{1}{p_{i}}\right] L_{i}\right) / L_{i}$ where the $i$-th summand is a divisible $p_{i}$-group. This shows that $R\left(\tau_{i}\right)=Z\left[\frac{1}{p_{i}}\right] L_{i}$ for all $i<\omega$. Now let $\varphi \in \operatorname{End}_{\mathrm{Z}}(R)$. Then $\varphi\left(Z_{Z}\left[\frac{1}{p_{i}}\right] L_{i}\right)=\varphi\left(R\left(\tau_{i}\right)\right) \subseteq R\left(\tau_{i}\right)=Z_{\mathbb{Z}}\left[\frac{1}{p_{i}}\right] L_{i}$. Let $\psi \in$ $\operatorname{End}_{\mathrm{Q}}(\mathrm{Q}[X])$ be the unique homomorphism induced by $\varphi$. Then $\psi\left(\mathrm{Q} L_{i}\right) \subseteq$ $\mathrm{Q} L_{i}$ for all $i<\omega$ and Theorem 2 supplies some $d \in \mathrm{Q}[X]$ with $\psi=d \cdot$. Since $d=\psi(1)=\varphi(1) \in R$, we have $\varphi=d$. for some $d \in R$, i.e. $R$ is an E-ring.

Let us call such a ring $R$ an (GHS)-E-ring with prime number set $P$.

LEMMA 2. Let $B$ be a torsion-free abelian group and $R, \tau_{i}$ as above. If $B\left(\tau_{i}\right)=\{0\}$, then $(B \otimes R)\left(\tau_{i}\right)=B \otimes R\left(\tau_{i}\right)$. (Here " $\otimes$ " $i$ s understood to mean “ $\otimes z$ ")

Proof. Consider the short exact sequence $0 \rightarrow Z[X] \rightarrow R \rightarrow$ $R / Z[X] \cong \bigoplus_{i<\omega}\left(\left(Z\left[\frac{1}{p_{i}}\right] L_{i}\right) / L_{i}\right) \rightarrow 0$, which gives rise to the sequence $0 \rightarrow B \otimes Z^{i<\omega}[X] \rightarrow B \otimes R \rightarrow \bigoplus_{i<\omega} B \otimes\left(\left(Z\left[\frac{1}{p_{i}}\right] L_{i}\right) / L_{i}\right) \rightarrow 0$. Note that $Z[X]$ is a free abelian group and thus $B \otimes \mathbb{Z}[X]$ is isomorphic to a direct sum of copies of $B$ and thus has no elements of infinite $p_{i}$-height. Let $x \in(B \otimes R)\left(\tau_{i}\right)$, i.e. $x$ has infinite $p_{i}$-height in $B \otimes R$. We may assume that $x \in B \otimes \mathbb{Z}[X]$. Then $\mathbb{Z}\left[\frac{1}{p_{i}}\right] x \subseteq B \otimes R$ and $\left(\left(Z_{[}\left[\frac{1}{p_{i}}\right] x+B\right) \otimes \mathbb{Z}[X]\right) /$ $(B \otimes \mathbb{Z}[X])$ is a $p_{i}$-torsion group. We infer that $\mathbb{Z}\left[\frac{1}{p_{i}}\right] x \subseteq B \otimes(\mathbb{Z}[X]+$ $\left.Z_{L}\left[\frac{1}{p_{i}}\right] L_{i}\right)=B \otimes\left(T_{i} \oplus Z_{[}\left[\frac{1}{p_{i}}\right] L_{i}\right)$ with $T_{i}$ free abelian. It follows that $Z\left[\frac{1}{p_{i}}\right] x \subseteq B \otimes Z\left[\frac{1}{p_{i}}\right] L_{i}$ and the claim follows. 
We are now ready for the following:

THEOREM 3. Let B be a torsion-free abelian group and $P$ an infinite set of prime integers such that $B$ is $p$-reduced for all $p \in P$. Let $R$ be a (GHS)$E$-ring with prime number set $P$. Then the natural map $\alpha: B \rightarrow B \otimes_{\mathrm{Z}} R$ is an absolute localization of $B$.

Proof. Note that $\alpha(x)=x \otimes 1$ for all $x \in B$. It follows from Lemma 2 that $B \otimes R$ is an $\mathrm{E}(\mathrm{R})$-module. By Proposition 1.2 in [6] we obtain that $\operatorname{End}_{Z}(B \otimes R)=\operatorname{End}_{R}(B \otimes R)$. By Proposition 1.1 in [6] the map $\alpha$ is a localization of $B$.

\section{Examples}

The following is a well-known and highly instructive example.

ExAMPLE 1. Even if $A$ is p-reduced, the tensor product $A \otimes A$ might not be p-reduced:

Let $p$ be prime, $J_{p}$ the ring of $p$-adic numbers, $Z_{p}$ the ring of integers localized at $p$, and $\pi \in J_{p}-Z_{p}$, a unit. Thus

$$
\pi=\lim _{n \rightarrow \infty} z_{n},
$$

$z_{n} \in Z_{p}$, in the $p$-adic topology with $p \nmid z_{n}$ and $p^{n} \mid\left(\pi-z_{n}\right)$ in $J_{p}$.

Let $A=\langle 1, \pi\rangle_{*} \subseteq J_{p}$, the pure subgroup of $J_{p}$ generated by 1 and $\pi$. Then $A \otimes A=\langle 1 \otimes 1,1 \otimes \pi, \pi \otimes 1, \pi \otimes \pi\rangle_{*}$ is a pure subgroup of rank 4 of $J_{p} \otimes J_{p}$.

Since $\pi \otimes \pi-\pi \otimes z_{n}=\pi \otimes\left(\pi-z_{n}\right)$ we have $p^{n} \mid\left(\pi \otimes \pi-\pi \otimes z_{n}\right)$. Similarly $p^{n} \mid\left(\pi \otimes \pi-z_{n} \otimes \pi\right)$. Hence $p^{n} \mid\left[\left(\pi \otimes \pi-\pi \otimes z_{n}\right)-\left(\pi \otimes \pi-z_{n} \otimes \pi\right)\right]=$ $(1 \otimes \pi-\pi \otimes 1) z_{n}$. Then there must exist $\alpha_{n}$ and $\beta_{n}$ in the integers such that $1=z_{n} \alpha_{n}+p^{n} \beta_{n}$. Hence $p^{n} \mid(1 \otimes \pi-\pi \otimes 1) z_{n} \alpha_{n}=(1 \otimes \pi-\pi \otimes 1)\left(1-p^{n} \beta_{n}\right)$ which implies $p^{n} \mid(1 \otimes \pi-\pi \otimes 1)$ for all $n<\omega$.

Since $A$ is a $Z_{p}$-module this means $0 \neq 1 \otimes \pi-\pi \otimes 1$ is a divisible element. Hence $A \otimes A$ is not reduced.

EXAMPLE 2. Even if $A$ is strongly indecomposable, the tensor product $A \otimes A$ might be completely decomposable:

Given distinct primes $\mathrm{p}, \mathrm{q}, \mathrm{r}$, define $A=e_{1} \mathrm{Z}\left[\frac{1}{p}\right] \oplus e_{2} \mathrm{Z}\left[\frac{1}{q}\right]+\left(e_{1}+e_{2}\right) Z\left[\frac{1}{r}\right]$. 
Then $A$ is strongly indecomposable of rank 2 with $\operatorname{End}(A)=Z$. We have

$$
\begin{aligned}
& A \otimes A=b_{1} Z_{[}\left[\frac{1}{p}\right] \oplus b_{2} Z_{1}\left[\frac{1}{p q}\right] \oplus b_{3} Z\left[\frac{1}{p q}\right] \oplus b_{4} Z\left[\frac{1}{q}\right]+ \\
& +\left(b_{1}+b_{3}\right) Z\left[\frac{1}{r p}\right]+\left(b_{2}+b_{4}\right) Z\left[\frac{1}{r q}\right]+\left(b_{1}+b_{2}\right) Z\left[\frac{1}{p r}\right]+ \\
& +\left(b_{3}+b_{4}\right) Z\left[\frac{1}{q r}\right]+\left(b_{1}+b_{2}+b_{3}+b_{4}\right) Z\left[\frac{1}{r}\right]
\end{aligned}
$$

where $b_{1}=e_{1} \otimes e_{1}, b_{2}=e_{1} \otimes e_{2}, b_{3}=e_{2} \otimes e_{1}, b_{4}=e_{2} \otimes e_{2}$.

Define $\rho$ by $\rho\left(b_{1}\right)=b_{1}+b_{2}$, and $\rho\left(b_{i}\right)=0$ for $2 \leq i \leq 4$. Then

$$
\begin{aligned}
& \rho(A \otimes A)=\left(b_{1}+b_{2}\right) Z\left[\frac{1}{p}\right]+\left(b_{1}+b_{2}\right) Z\left[\frac{1}{r p}\right]+ \\
& +\left(b_{1}+b_{2}\right) Z\left[\frac{1}{p r}\right]+\left(b_{1}+b_{2}\right) Z\left[\frac{1}{r}\right]=\left(b_{1}+b_{2}\right) Z\left[\frac{1}{p r}\right] .
\end{aligned}
$$

and thus $\rho(A \otimes A) \subseteq A \otimes A$, i.e. $\rho \in \operatorname{End}(A \otimes A)$ with $\rho^{2}=\rho$.

Define $\sigma$ by $\sigma\left(b_{4}\right)=b_{3}+b_{4}$, and $\sigma\left(b_{i}\right)=0$ for $1 \leq i \leq 3$. Then

$$
\begin{aligned}
& \sigma(A \otimes A)=\left(b_{3}+b_{4}\right) Z\left[\frac{1}{q r}\right]+\left(b_{3}+b_{4}\right) Z\left[\frac{1}{q r}\right]+ \\
& +\left(b_{3}+b_{4}\right) Z\left[\frac{1}{q}\right]+\left(b_{3}+b_{4}\right) Z\left[\frac{1}{r}\right]= \\
& =\left(b_{3}+b_{4}\right) Z\left[\frac{1}{q r}\right] \subseteq A \otimes A \text { and thus } \\
& \sigma \in \operatorname{End}(A \otimes A), \sigma^{2}=\sigma . \sigma \circ \rho=\rho \circ \sigma=0 .
\end{aligned}
$$

Note that $(1-\rho-\sigma)\left(b_{i}\right)=\left\{\begin{array}{cc}-b_{2} & \text { for } i=1 \\ b_{2} & \text { for } i=2 \\ b_{3} & \text { for } i=3 \\ -b_{3} & \text { for } i=4\end{array}\right.$

and $(1-\rho-\sigma)(A \otimes A)=-b_{2} Z_{4}\left[\frac{1}{p}\right]+b_{2} Z\left[\frac{1}{p q}\right]+b_{3} Z\left[\frac{1}{p q}\right]-b_{3} Z\left[\frac{1}{q}\right]+$

$$
\begin{aligned}
& +\left(-b_{2}+b_{3}\right) Z\left[\frac{1}{r p}\right]+\left(b_{2}-b_{3}\right) Z\left[\frac{1}{r q}\right]+0+0+0= \\
& =b_{2} Z\left[\frac{1}{p q}\right]+b_{3} Z\left[\frac{1}{p q}\right]+\left(b_{2}-b_{3}\right) Z\left[\frac{1}{p q r}\right]=: C .
\end{aligned}
$$


Observe that $C=\left(b_{2}-b_{3}\right) Z\left[\frac{1}{p q r}\right] \oplus b_{2} Z\left[\frac{1}{p q}\right]$, and thus

$A \otimes A=\underbrace{\left(b_{1}+b_{2}\right) Z\left[\frac{1}{p r}\right]}_{i m(\rho)} \oplus \underbrace{\left(b_{3}+b_{4}\right) Z\left[\frac{1}{q r}\right]}_{i m(\sigma)} \oplus \underbrace{\left(b_{2}-b_{3}\right) Z\left[\frac{1}{p q r}\right] \oplus b_{2} Z\left[\frac{1}{p q}\right]}_{i m(1-\rho-\sigma)=C}$ is completely decomposable.

\section{Torsion-free Abelian Groups of Finite Rank with Small p-rank}

Again, let $J_{p}$ denote the ring of $p$-adic integers and " $\otimes$ " means " $\otimes \mathrm{Z}$ ".

We view $J_{p} \otimes J_{p}$ as a right $J_{p}$-module. (Of course, $J_{p} \otimes J_{p}$ is also a left $J_{p}$-module, but we need to pick a side.)

Let $D$ denote the divisible part of the torsion-free group $J_{p} \otimes J_{p}$. There is a natural (surjective) map $\mu: J_{p} \otimes J_{p} \rightarrow J_{p}$ with $\mu(a \otimes b)=a b$. Since $J_{p}$ is $p$-reduced, we have $D \subseteq \operatorname{ker}(\mu)$.

Let $w=\sum_{1 \leq i \leq n} a_{i} \otimes b_{i}=\sum_{1 \leq i \leq n}\left(a_{i} \otimes 1\right) b_{i} \in \operatorname{ker}(\mu)$ and $v=\sum_{1 \leq i \leq n}\left(1 \otimes a_{i}-\right.$ $\left.a_{i} \otimes 1\right) b_{i}$. Then $v \in D$ as shown in Example 1. Note that $0=1 \otimes \mu(w)=$ $1 \otimes\left(\sum_{1 \leq i \leq n} a_{i} b_{i}\right)=\sum_{1 \leq i \leq n}\left(1 \otimes a_{i}\right) b_{i}=w+v$. This shows that $w=-v \in D$ and thus $D=\operatorname{ker}(\mu)$.

We record this as:

REMARK 2. We have $J_{p} \otimes J_{p}=D \oplus\left(1 \otimes J_{p}\right)$ and $\mu: J_{p} \otimes J_{p} \rightarrow J_{p}$ is a surjective homomorphism such that $D=\operatorname{ker}(\mu)$ is the divisible part of $J_{p} \otimes J_{p}$.

Let $G$ be a torsion-free group and $Z_{p}$ the ring of integers localized at the prime $p$. Then each $y \in G \otimes Z_{p}$ has the form $y=a \otimes \frac{1}{n}$ for some $a \in G$ and integer $n$ relatively prime to $p$. An easy argument shows that $G$ is $p$ reduced if and only if $G \otimes Z_{p}$ is $p$-reduced.

Let $A$ be a torsion-free, $p$-reduced group of, say, rank 2 and $p$-rank 1 . It is easy to see that there exists a $p$-pure subgroup $A^{\prime}$ of $J_{p}$ such that $1 \in A^{\prime}$ and $A \cong A^{\prime}$.

Notation 1. Let $G$ be a p-reduced, torsion-free $Z_{p}$-module of finite rank $n+m$ and $n=r_{p}(G)=\operatorname{dim}_{\mathrm{Z}_{/} \mathrm{ZZ}}(G / p G)$ for some prime $p$. Then $G$ 
can be represented as a pure subgroup of $\bigoplus e_{i} J_{p}$ generated by $B=\bigoplus_{1 \leq i \leq n} e_{i} Z_{p}$ together with elements $u_{j}=\sum_{1 \leq i \leq n} e_{i} \pi_{i j}$ for $1 \leq j \leq m$ and $\Pi=\left[\pi_{i j}\right] \in \operatorname{Mat}_{n \times m}\left(J_{p}\right)$. We call $\Pi$ a representing matrix for $G$. Of course, the matrix $\Pi$ is not uniquely determined by $G$. Moreover, we may, and will, make the convention that $\pi_{i j}=0$ whenever $\pi_{i j} \in Z_{p}$.

Fomin [8] has shown that if the entries of the matrix $\Pi$ are algebraically independent (over $Z_{p}$ ) and $G \otimes G$ is not $p$-reduced but $G$ is $p$-reduced, then $r_{p}(G)=1$. The following example shows that this rather strong hypothesis on $\Pi$ is needed:

EXAMPLE 3. There exists a p-reduced torsion-free $Z_{p}$-module $G$ such that $\operatorname{rank}(G)=4, r_{p}(G)=2$ and $G \otimes G$ is not p-reduced. Moreover, $G$ does not contain a pure subgroup $A$ with $r_{p}(A)=1$ and $\operatorname{rank}(A)=2$.

Proof. Pick an odd prime $p$ and $\alpha, \beta, \gamma \in \mathbb{N}$. such that $\sqrt{\alpha}, \sqrt{\beta}$, $\sqrt{\gamma} \in J_{p}-Z_{p}$ and the field extensions $\mathrm{Q}[\sqrt{\beta}, \sqrt{\alpha}]$ and $\mathrm{Q}[\sqrt{\beta}, \sqrt{\gamma}]$ have dimension 4 over $\mathrm{Q}$ with $\sqrt{\gamma} \notin \mathrm{Q}[\sqrt{\beta}, \sqrt{\alpha}]$. Let $u=e_{1} \sqrt{\alpha} \otimes e_{2} \sqrt{\beta \gamma}$ and $v=e_{1} \sqrt{\alpha \beta} \otimes e_{2} \sqrt{\gamma}$. Let $G=\left\langle e_{1} z_{p} \oplus e_{2} Z_{p}, u, v\right\rangle_{*} \subset e_{1} J_{p} \oplus e_{2} J_{p}$. Let $w=$ $e_{1} \otimes e_{1} \alpha(\beta-1)+e_{2} \otimes e_{2} \gamma(1-\beta)+u \otimes u-v \otimes v \in G \otimes G \subset \underset{1 \leq i, j \leq 2}{\bigoplus_{1} J_{p}\left(e_{i} \otimes e_{j}\right) J_{p} .}$ We claim that

$w$ is a $p$-divisible element of $G$ :

Note that $w=\left[e_{1} \otimes e_{1} \alpha(\beta-1)+\sqrt{\alpha} e_{1} \otimes e_{1} \sqrt{\alpha}-\sqrt{\alpha \beta} e_{1} \otimes e_{1} \sqrt{\alpha \beta}\right]+$ $+\left[\sqrt{\alpha} e_{1} \otimes e_{2} \sqrt{\beta \gamma}-\sqrt{\alpha \beta} e_{1} \otimes e_{2} \sqrt{\gamma}\right]+$ $+\left[\sqrt{\beta \gamma} e_{2} \otimes e_{1} \sqrt{\alpha}-\sqrt{\gamma} e_{2} \otimes e_{1} \sqrt{\alpha \beta}\right]+$ $+\left[e_{2} \otimes e_{2} \gamma(1-\beta)+\sqrt{\beta \gamma} e_{2} \otimes e_{2} \sqrt{\beta \gamma}-\sqrt{\gamma} e_{2} \otimes \sqrt{\gamma} e_{2}\right]$.

Each term in a square bracket is in the divisible part of $J_{p} e_{i} \otimes e_{j} J_{p}$ and thus $w \neq 0$ is a divisible element of $G \otimes G$.

Now let $0 \neq g=e_{1} a_{1}+e_{2} a_{2}+u b+v c \in G$. We claim that $g \pi \notin G$ for all $\pi \in J_{p}-Z_{p}$. This allows us to infer that $G$ has no pure subgroup $A$ with $\operatorname{rank}(A)=2$ and $p$-rank $r_{p}(A)=1$.

Let $g^{\prime}=e_{1} a_{1}^{\prime}+e_{2} a_{2}^{\prime}+u b^{\prime}+v c^{\prime} \in G$ such that $g \pi=g^{\prime}$.

W.l.o.g. we may assume that all coefficients are integers. Using matrix notation, we get 
$\left[\begin{array}{l}a_{1}+b \sqrt{\alpha}+c \sqrt{\alpha \beta} \\ a_{2}+b \sqrt{\beta \gamma}+c \sqrt{\gamma}\end{array}\right] \pi=\left[\begin{array}{l}a_{1}^{\prime}+b^{\prime} \sqrt{\alpha}+c^{\prime} \sqrt{\alpha \beta} \\ a_{2}^{\prime}+b^{\prime} \sqrt{\beta \gamma}+c^{\prime} \sqrt{\gamma}\end{array}\right]$.

Case 1: $a_{1}+b \sqrt{\alpha}+c \sqrt{\alpha \beta} \neq 0 \neq a_{2}+b \sqrt{\beta \gamma}+c \sqrt{\gamma}$.

In this case we have $\pi \in \mathbb{Q}[\sqrt{\alpha}, \sqrt{\beta}] \cap \mathbb{Q}[\sqrt{\beta}, \sqrt{\gamma}]=\mathrm{Q}[\sqrt{\beta}]$ and w.l.o.g. we may assume that $\pi=\sqrt{\beta}$ and we get

$$
\begin{aligned}
& {\left[\begin{array}{l}
a_{1}+b \sqrt{\alpha}+c \sqrt{\alpha \beta} \\
a_{2}+b \sqrt{\beta \gamma}+c \sqrt{\gamma}
\end{array}\right] \sqrt{\beta}=} \\
& {\left[\begin{array}{c}
\sqrt{\beta} a_{1}+b \sqrt{\alpha \beta}+c \beta \sqrt{\alpha} \\
\sqrt{\beta} a_{2}+c \sqrt{\beta \gamma}+b \beta \sqrt{\gamma}
\end{array}\right]=\left[\begin{array}{l}
a_{1}^{\prime}+b^{\prime} \sqrt{\alpha}+c^{\prime} \sqrt{\alpha \beta} \\
a_{2}^{\prime}+b^{\prime} \sqrt{\beta \gamma}+c^{\prime} \sqrt{\gamma}
\end{array}\right]}
\end{aligned}
$$

and we infer the equations:

$a_{1}=0=a_{1}^{\prime}, b=c^{\prime}, c \beta=b^{\prime}$ as well as

$a_{2}=0=a_{2}^{\prime}, c=b^{\prime}, b \beta=c^{\prime}$ and thus $\beta b=b$ and $c \beta=c$. We now have that $b=0=c$ and $g=0$, a contradiction.

Case 2: $a_{1}+b \sqrt{\alpha}+c \sqrt{\alpha \beta}=0$ but $a_{2}+b \sqrt{\beta \gamma}+c \sqrt{\gamma} \neq 0$ (or vice versa).

In this case we have that $a_{1}^{\prime}=b^{\prime}=c^{\prime}=0=a_{1}=b=c$ and thus $a_{2} \pi=a_{2}^{\prime}$, a contradiction to $\pi \notin Z_{p}$.

A possible choice for $p, \alpha, \beta, \gamma$ satisfying our hypotheses would be $p=19, \alpha=7, \beta=11$ and $\gamma=17$, because these are distinct primes and $8^{2} \equiv 7 \bmod 19,7^{2} \equiv 11 \bmod 19$ and $6^{2} \equiv 17 \bmod 19$. Therefore, by Hensel's Lemma, we have that $\sqrt{7}, \sqrt{11}, \sqrt{17} \in J_{19}$.

Next we will introduce a quasi-isomorphism invariant for our group $G$.

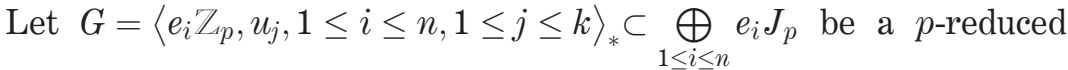
group of rank $n+k$ and $r_{p}(G)=n$ where $u_{j}=\sum_{1 \leq i \leq n} e_{i} \pi_{i j}$ with $\pi_{i j} \in J_{p}$. Let $G^{\prime}=\left\langle e_{i}^{\prime} Z_{p}, u_{j}^{\prime}, 1 \leq i \leq n^{\prime}, 1 \leq j \leq k^{\prime}\right\rangle_{*} \subset \bigoplus_{1 \leq i \leq n} e_{i}^{\prime} J_{p}$ be another such group with $u_{j}^{\prime}=\sum_{1 \leq i \leq n^{\prime}} e_{i}^{\prime} \pi_{i j}^{\prime}$ for $1 \leq j \leq k^{\prime}$.

Assume that for all $\beta, 1 \leq \beta \leq n^{\prime}$, the sets $\Pi_{\beta}=\left\{1, \pi_{\beta j}^{\prime}: 1 \leq j \leq k^{\prime}\right\}$ are linearly independent over the field extension $K=\mathbb{Q}\left(\pi_{i j}, 1 \leq i \leq n, 1 \leq j \leq k\right)$. Then $G \otimes G^{\prime}$ is $p$-reduced:

We have $G \otimes G^{\prime}=\bigoplus_{i, j} e_{i} \otimes e_{j}^{\prime} Z_{p}+\sum_{i, j} e_{i} \otimes u_{j}^{\prime} Z_{p}+\sum_{i, j} u_{i} \otimes e_{j}^{\prime} Z_{p}+\sum_{i, j} u_{i} \otimes u_{j}^{\prime} Z_{p}$. Let $y=\sum_{1 \leq i, j \leq n} e_{i} \otimes e_{j}^{\prime} a_{i j}+\sum_{i, j} e_{i} \otimes u_{j}^{\prime} b_{i j}+\sum_{i, j} u_{i} \otimes e_{j}^{\prime} c_{i j}+\sum_{i, j} u_{i} \otimes u_{j}^{\prime} d_{i j} \in G \otimes G^{\prime}$. 
Note that the $e_{\alpha} \otimes e_{\beta}^{\prime}$ entry of $y$ is

$y_{\alpha \beta}=e_{\alpha} \otimes e_{\beta}^{\prime} a_{\alpha \beta}+\sum_{j} e_{\alpha} \otimes e_{\beta}^{\prime} \pi_{\beta j}^{\prime} b_{\alpha j}+\sum_{i} \pi_{\alpha i} e_{\alpha} \otimes e_{\beta}^{\prime} c_{i \beta}+\sum_{i, j} \pi_{\alpha i}\left(e_{\alpha} \otimes e_{\beta}^{\prime}\right) \pi_{\beta j}^{\prime} d_{i j}$

If $y$ is a divisible element, then, by Remark 2 , we have

$$
\begin{aligned}
0=\mu\left(y_{\alpha \beta}\right)=a_{\alpha \beta} & +\sum_{j} \pi_{\beta j}^{\prime} b_{\alpha j}+\sum_{i} \pi_{\alpha i} c_{i \beta}+\sum_{i, j} \pi_{\alpha i} \pi_{\beta j}^{\prime} d_{i j}= \\
= & \left(a_{\alpha \beta}+\sum_{i} \pi_{\alpha i} c_{i \beta}\right)+\sum_{j} \pi_{\beta j}^{\prime}\left(b_{\alpha j}+\sum_{i} \pi_{\alpha i} d_{i j}\right) \text { for all } \alpha, \beta .
\end{aligned}
$$

By our hypothesis that $\left\{1, \pi_{\beta j}^{\prime}: 1 \leq j \leq k^{\prime}\right\}$ is linearly independent over the field extension $K=\mathrm{Q}\left(\pi_{i j}, 1 \leq i \leq n, 1 \leq j \leq k\right)$, we infer that $a_{\alpha \beta}+\sum_{i} \pi_{\alpha i} c_{i \beta}=0$ and it follows that $\sum_{i} u_{i} c_{i \beta} \in \underset{1 \leq j \leq n}{\bigoplus} e_{j} Z_{p}$. This is a contradiction to $n+k=\operatorname{rank}(G)$ unless all $a_{\alpha \beta}=0=c_{i \beta}$.

We infer that $\sum_{j} \pi_{\beta j}^{\prime}\left(b_{\alpha j}+\sum_{i} \pi_{\alpha i} d_{i j}\right)=0$ for all $\alpha, \beta$ and thus $b_{\alpha j}+\sum_{i} \pi_{\alpha i} d_{i j}=0$ for all $\alpha, j$. Again, it follows that $\sum_{i} u_{i} d_{i j} \in \underset{1 \leq \gamma \leq n}{\bigoplus} e_{\gamma} Z_{p}$ and thus $d_{i j}=0$ for all $i, j$. Now we infer that $b_{\alpha j}=0$ for all $\alpha, j$ and we have that $y=0$.

Proposition 8. Let $G=\left\langle e_{i} Z_{p}, u_{j}, 1 \leq i \leq n, 1 \leq j \leq k\right\rangle_{*} \subset \underset{1 \leq i \leq n}{\bigoplus} e_{i} J_{p}$ be a p-reduced group of rank $n+k$ and $r_{p}(G)=n$ where $u_{j}=\sum_{1 \leq i \leq n} e_{i} \pi_{i j}$ with $\pi_{i j} \in J_{p}$. Let $G^{\prime}=\left\langle e_{i}^{\prime} Z_{p}, u_{j}^{\prime}, 1 \leq i \leq n, 1 \leq j \leq k\right\rangle_{*} \subset \bigoplus_{1 \leq i \leq n} e_{i} J_{p}$ be another such group with $u_{j}^{\prime}=\sum_{1 \leq i \leq n} e_{i} \pi_{i j}^{\prime}$. Let $K\left(K^{\prime}\right)$ be the field extension of $\mathrm{Q}$ generated by the elements $\pi_{i j}\left(\pi_{i j}^{\prime}\right)$.

If $G$ is quasi-isomorphic to $G^{\prime}$, then $K=K^{\prime}$.

Proof. Note that $G=\left\langle\left(\bigoplus_{1 \leq i \leq n} e_{i} Z_{p}\right) \oplus\left(\bigoplus_{1 \leq i \leq k} u_{i} Z_{p}\right)\right\rangle_{*} \subset \bigoplus_{1 \leq i \leq n} e_{i} J_{p}$ where $u_{i}=\sum_{1 \leq j \leq n} e_{j} \pi_{j i}$ for some $\pi_{j i} \in J_{p}$. Put $\Pi=\left[\pi_{j i}\right] \in \operatorname{Mat}_{n \times k}\left(J_{p}\right)$.

Let $\varphi: G \rightarrow G^{\prime}$ be a quasi-isomorphism. Then there exist matrices $A=\left[a_{i j}\right] \in \operatorname{Mat}_{n \times n}(\mathbb{Q}), B=\left[b_{i j}\right] \in \operatorname{Mat}_{k \times n}(\mathbb{Q})$ such that

$$
\varphi\left(e_{i}\right)=\sum_{j} e_{j}^{\prime} a_{j i}+\sum_{j} u_{j}^{\prime} b_{j i} \text {. Now }
$$




$$
\begin{aligned}
& \varphi\left(u_{i}\right)=\sum_{j} \varphi\left(e_{j}\right) \pi_{j i}=\sum_{j}\left(\sum_{\alpha} e_{\alpha}^{\prime} a_{\alpha j}+\sum_{\beta} u_{\beta}^{\prime} b_{\beta j}\right) \pi_{j i}= \\
& =\sum_{j}\left(\sum_{\alpha} e_{\alpha}^{\prime} a_{\alpha j}+\sum_{\beta} \sum_{\alpha}\left(e_{\alpha}^{\prime} \pi_{\alpha \beta}^{\prime}\right) b_{\beta j}\right) \pi_{j i}= \\
& =\sum_{\alpha} e_{\alpha}^{\prime}\left(\sum_{j}\left(a_{\alpha j} \pi_{j i}+\sum_{\beta} \pi_{\alpha \beta}^{\prime} b_{\beta j} \pi_{j i}\right)\right)= \\
& =\sum_{\alpha} e_{\alpha}^{\prime}\left([A \Pi]_{\alpha i}+\left[\Pi^{\prime} B \Pi\right]_{\alpha i}\right) .
\end{aligned}
$$

Since $\varphi\left(u_{i}\right) \in G^{\prime}$ for all $1 \leq i \leq k$, we have that

$$
\begin{aligned}
& \varphi\left(u_{i}\right)=\sum_{j} e_{j}^{\prime} x_{j i}+\sum_{j} u_{j}^{\prime} y_{j i}=\sum_{\alpha} e_{\alpha}^{\prime} x_{\alpha i}+\sum_{j}\left(\sum_{\alpha} e_{\alpha}^{\prime} \pi_{\alpha j}^{\prime}\right) y_{j i}= \\
& =\sum_{\alpha} e_{\alpha}^{\prime}\left(x_{\alpha i}+\sum_{j} \pi_{\alpha j}^{\prime} y_{j i}\right) . \text { Let } X=\left[x_{\alpha i}\right] \in \operatorname{Mat}_{n \times k}(\mathrm{Q}) \text { and } \\
& Y=\left[y_{\alpha i}\right] \in \operatorname{Mat}_{k \times k}(\mathrm{Q}) . \text { We now have a matrix equation }
\end{aligned}
$$

$A \Pi+\Pi^{\prime} B \Pi=X+\Pi^{\prime} Y$ of $n \times k$ matrices, which is equivalent to

$\Pi^{\prime}(B \Pi-Y)=X-A \Pi$ where $B \Pi-Y$ is a square $k \times k$ matrix.

Note that

$$
\varphi\left(e_{i}\right)=\sum_{j} e_{j}^{\prime} a_{j i}+\sum_{j} u_{j}^{\prime} b_{j i}=\sum_{\alpha} e_{\alpha}^{\prime} a_{\alpha i}+\sum_{j} \sum_{\alpha} e_{\alpha}^{\prime} \pi_{\alpha j}^{\prime} b_{j i}=\sum_{\alpha} e_{\alpha}^{\prime}\left(A+\Pi^{\prime} B\right)_{\alpha i} .
$$

Since $\varphi$ is injective, the square matrix $\left[\begin{array}{cc}A & X \\ B & Y\end{array}\right] \in \operatorname{Mat}_{(n+k) \times(n+k)}(\mathrm{Q})$ is invertible.

Assume that there is some $T \in \operatorname{Mat}_{k \times k}\left(J_{p}\right)$ such that $(B \Pi-Y) T=0$. Then $0=\Pi^{\prime}(B \Pi-Y) T=(X-A \Pi) T$.

This implies that $\left[\begin{array}{ll}A & X \\ B & Y\end{array}\right]\left[\begin{array}{c}\Pi T \\ -T\end{array}\right]=\left[\begin{array}{c}A \Pi T-X T \\ B \Pi T-Y T\end{array}\right]=\left[\begin{array}{l}0 \\ 0\end{array}\right]$. Since $\left[\begin{array}{ll}A & X \\ B & Y\end{array}\right]$ is invertible, we infer that $T=0$. It follows that $B \Pi-Y$ is invertible.

Let $K=\mathrm{Q}\left(\pi_{i j}, 1 \leq i \leq n, 1 \leq j \leq k\right)$ and $K^{\prime}=\mathrm{Q}\left(\pi_{i j}^{\prime}, 1 \leq i \leq n, 1 \leq j \leq k\right)$. Then $B \Pi-Y \in \operatorname{Mat}_{m \times m}(K)$ and so is $(B \Pi-Y)^{-1}$, which implies $\Pi^{\prime} \in$ $\operatorname{Mat}_{k \times k}(K)$ and thus $K^{\prime} \subseteq K$. By symmetry it follows that $K=K^{\prime}$.

Let $A$ be an E-ring. Let $\varphi \in H o m(A \otimes A, A)$. The group $A \otimes A$ contains subgroups of the form $A \otimes b$ and $a \otimes A$. There exists a surjective map $i_{b}: A \rightarrow A \otimes b$ with $i_{b}(x)=x \otimes b$ for all $x \in A$. Then $\psi_{b}=\varphi \circ i_{b}: A \rightarrow A$ 
and since $A$ is an E-ring, there is some $\sigma_{b} \in A$ such that $\psi_{b}(x)=$ $\varphi(x \otimes b)=x \sigma_{b}$ for all $x \in A$. By a similar argument, there are elements $\pi_{a} \in A$ such that $\varphi(a \otimes y)=y \pi_{a}$ for all $y, a \in A$.

Note that $\varphi(a \otimes b)=b \pi_{a}=\sigma_{b} a$. It follows that $\sigma_{1}=\pi_{1}$. For $a=1$ we get $\sigma_{b}=b \pi_{1}$ and thus $\varphi(a \otimes b)=a b \pi_{1}$ for all $a, b \in A$.

We conclude that $\varphi=\pi_{1} \mu$ where $\mu \in \operatorname{Hom}(A \otimes A, A)$ is the map with $\mu(a \otimes b)=a b$ for all $a, b \in A$. We have shown:

Proposition 9. If $A$ is an E-ring, then the map $\mu: A \otimes A \rightarrow A$ with $\mu(a \otimes b)=a b$ for all $a, b \in A$ is a localization.

\section{Zero Product Determined Algebras}

The following definition can be found in [4] and elsewhere:

Definition 4. Let $A$ be an algebra over the commutative ring $C$. Then $A$ is called zero product determined if for any $C$-module $X$ and every $C$ bilinear map $\left\langle_{-} \mid{ }_{-}\right\rangle: A \times A \rightarrow X$ the following holds:

If for all $a, b \in A, a b=0$ implies $\langle a \mid b\rangle=0$, then there exists some $T \in \mathrm{Hom}_{C}\left(A^{2}, X\right)$ with $\langle x \mid y\rangle=T(x y)$ for all $x, y \in A$. As usual $A^{2}$ denotes the $C$-submodule of $A$ generated by the set of products $x y$ for $x, y \in A$.

REMARK 3. Let $C$ be a commutative ring and $X$ some $C$-module. We will always assume that $X$ is a $C$-bimodule with $s x=x$ sor all $x \in X$ and all $s \in C$. Let $X, Y, W$ be $C$-modules. Then each $C$-linear map $\left\langle_{-} \mid{ }_{-}\right\rangle: X \times Y \rightarrow W$ is automatically a middle linear map, i.e. $\langle x s \mid y\rangle=$ $\langle x \mid s y\rangle$ for all $x \in X, y \in Y$ and $s \in C$. This means that $\left\langle_{-} \mid{ }_{-}\right\rangle$factors through $X \otimes_{C} Y$. We will write " $\otimes$ " instead of “ $\otimes_{C}$ ".

Proposition 10. Let $A$ be a C-algebra. Consider the maps $A \times A \stackrel{\otimes}{\rightarrow} A \otimes A \stackrel{\mu}{\rightarrow} A^{2}=\operatorname{span}_{C}\{x y: x, y \in A\}$ where $\mu(x \otimes y)=x y$.

The following are equivalent:

(a) Let $X$ be some $C$-module and $\theta \in H_{C}(A \otimes A, X)$. If $\operatorname{ker}(\mu \circ \otimes) \subseteq$ $\operatorname{ker}(\theta \circ \otimes)$, then there exists some $T \in \operatorname{Hom}_{C}\left(A^{2}, X\right)$ making the following diagram commutative:

$$
A \times A \stackrel{\otimes}{\rightarrow} A \otimes A \stackrel{\mu}{\rightarrow} A^{2}
$$

then $A=A^{2}$.

$$
\begin{aligned}
& \downarrow \theta \quad{ }^{T} \quad \text {. Note that if } 1 \in A \text {, } \\
& \text { X }
\end{aligned}
$$

(b) $A$ is zero product determined. 
(c) Let $X$ be some $C$-module and $\theta \in H_{C}(A \otimes A, X)$. If $\operatorname{ker}(\mu \circ \otimes) \subseteq$ $\operatorname{ker}(\theta \circ \otimes)$, then $\operatorname{ker}(\mu) \subseteq \operatorname{ker}(\theta)$.

Proof. Assume that (a) holds and let $\left\langle\left._{-}\right|_{-}\right\rangle: A \times A \rightarrow X$ be a $C$-bilinear map such that $\langle a \mid b\rangle=0$ whenever $a b=0$. Then there exists a unique $\theta \in H_{C}(A \otimes A, X)$ such that $\langle a \mid b\rangle=\theta(a \otimes b)$ for all $a, b \in A$. Thus $\theta(a \otimes b)=0$ whenever $a b=0$, i.e. $\operatorname{ker}(\mu \circ \otimes) \subseteq \operatorname{ker}(\theta \circ \otimes)$. By clause (a), there is some $T \in \operatorname{Hom}_{C}\left(A^{2}, X\right)$ such that $\theta=T \circ \mu$. It follows that $\langle a \mid b\rangle=(T \circ \mu)(a \otimes b)=T(a b)$ for all $a, b \in A$ and (b) holds.

For the converse, assume that (b) holds. With the notations in (a), define $\left\langle-\mid{ }_{-}\right\rangle: A \times A \rightarrow X$ by $\langle a \mid b\rangle=\theta(a \otimes b)$ for all $a, b \in A$. Since $\operatorname{ker}(\mu \circ \otimes) \subseteq$ $\operatorname{ker}(\theta \circ \otimes)$ we have that $a b=0$ implies $\langle a \mid b\rangle=0$ for all $a, b \in A$. By clause (b), there exists some $T \in \operatorname{Hom}_{C}\left(A^{2}, X\right)$ such that $\langle a \mid b\rangle=T(a b)$ for all $a, b \in A$. Therefore $\theta(a \otimes b)=\langle a \mid b\rangle=T(a b)=T(\mu(a \otimes b))$ for all $a, b \in A$. This shows that $\theta=T \circ \mu$ and (a) follows.

That (a) implies (c), is a trivial consequence of the commutative diagram in (a). For the converse, if $\operatorname{ker}(\mu) \subseteq \operatorname{ker}(\theta)$, the map $T$ may be defined by $T\left(\sum_{i} a_{i} b_{i}\right)=\theta\left(\sum_{i} a_{i} \otimes b_{i}\right)$, since $\sum_{i} a_{i} b_{i}=0$ means $\mu\left(\sum_{i} a_{i} \otimes b_{i}\right)=0$.

Note that the map $T$ in clause (a) is unique since the map $\mu$ is surjective.

Definition 5. Let $C$ be a commutative ring and $A$ a $C$-algebra and $X a$ $C$-module. Then $\operatorname{Hom}_{C}^{0}(A, X)=\left\{\theta \in \operatorname{Hom}_{C}(A \otimes A, X):\right.$ If $x, y \in A$ with $x y=0$, then $\theta(x \otimes y)=0\}$.

Then $A$ is zero product determined if and only if for any $\theta \in \operatorname{Hom}_{C}^{0}(A, X)$ there exists a (unique) $T \in \operatorname{Hom}_{C}\left(A^{2}, X\right)$ such that $\theta=T \circ \mu$.

Note that for any $T \in \operatorname{Hom}_{C}\left(A^{2}, X\right)$ we have that $T(\mu(x \otimes y))=$ $T(x y)=T(0)=0$ whenever $x y=0$, i.e. $T \circ \mu \in \operatorname{Hom}_{C}^{0}(A, X)$. This shows that

$\operatorname{Hom}_{C}\left(A^{2}, X\right) \stackrel{-\circ \mu}{\rightarrow} \operatorname{Hom}_{C}^{0}(A, X) \rightarrow 0$ is exact. We have shown:

Proposition 11. The $C$-algebra $A$ is zero product determined if and only if $\operatorname{Hom}_{C}\left(A^{2}, X\right) \cong \operatorname{Hom}_{C}^{0}(A, X)$ via the natural map _ $\circ \mu$.

Assume that $1 \in A$ has no zero divisors and is zero product determined. Then $\operatorname{Hom}_{C}^{0}(A, X)=\operatorname{Hom}_{C}(A \otimes A, X) \cong \operatorname{Hom}_{C}(A, X)$ via the natural map _ $\circ \mu$. 
Proposition 12. Let $C$ be an integral domain and $A$ a torsion-free $C$-algebra with $1 \in A$ and without zero divisors. Then $A$ is a zero product determined algebra if and only if $A$ is a subring of the field of fractions of $C$.

Proof. Note that $\operatorname{ker}(\mu \circ \otimes)=\{(a, 0): a \in A\} \cup\{(0, a): a \in A\} \subseteq$ $\operatorname{ker}(\theta \circ \otimes)$ for all $\theta \in \operatorname{Hom}_{C}(A \otimes A, X)$. Consider the identity map $i d: A \otimes A \rightarrow A \otimes A$. If $A$ is zero-product determined, then there exists a map $T: A \rightarrow A \otimes A$ such that $i d=T \circ \mu$ and thus $\mu$ is an isomorphism. This shows that the $C$-rank of $A$ is 1 and thus $A$ is a subring of the field of fractions of $C$. The converse is obvious.

COROLlary 6 . The ring $J_{p}$ of $p$-adic integers is not zero product determined.

This motivates a weaker condition:

DeFinition 6. Let $C$ be a commutative ring and $A$ a $C$-algebra. We call $A$ zero product self-determined, if $A$ has the property (a) of Proposition 11 with " $X$ " replaced by " $A$ ".

Theorem 4. Let $A$ be an integral domain. The following are equivalent:

(a) The ring $A$ is a zero product self-determined Z-algebra.

(b) The map $\mu: A \otimes A \rightarrow A$ is a (surjective) localization of $A \otimes A$.

(c) The ring $A$ is an $E$-ring.

Proof. Because of Proposition 9 and 10, we only have to show that (b) implies (c). To this end, pick any $\beta \in \operatorname{End}(A)$ and consider $i d_{A} \otimes \beta: A \otimes A \rightarrow$ $A \otimes A$. Then there exists some $T: A \rightarrow A$ with $\mu \circ\left(i d_{A} \otimes \beta\right)=T \circ \mu$ and thus $a \beta(b)=T(a b)$ for all $a, b \in A$. Let $a=1$. Then $\beta(b)=T(b)$ follows for all $b \in B$ and thus $a \beta(b)=\beta(a b)$ for all $a, b \in A$. Now, for $b=1$, we get $\beta(a)=a \beta(1)$ for all $a \in A$. Since $\beta \in \operatorname{End}(A)$ was arbitrary, it follows that $A$ is an E-ring. Note that the hypothesis that A has no zero divisors was not used in the previous argument.

We may use the previous argument and Proposition 9 to obtain the following characterization of E-rings: 
Corollary 7. Let $A$ be some ring (viewed as a Z-algebra). The following are equivalent:

(a) The natural map $\mu: A \otimes A \rightarrow A$ is a localization.

(b) The ring $A$ is an E-ring.

\section{REFERENCES}

[1] D. ARNOLD - B.O'BRIAN - J.D. REID, Quasipure injective and projective torsion-free abelian groups of finite rank. Proc. London Math. Soc. (3) 38 (1979), pp. 532-544.

[2] S. Breaz - G. Călugăreanu, Self-c-injective abelian groups. Rend. Sem. Mat. Univ. Padova 116 (2006), pp. 193-203.

[3] S. Breaz - P. Schultz, Dualities for self small groups. Proc. Amer. Math. Soc. 140 (2011), no. 1, pp. 69-82.

[4] M. BreŠAR - M. GraŠIČ - J.S. ORTEGA, Zero product determined matrix algebras. Lin. Alg. Appl. 430 (2009), pp. 1486-1498.

[5] M. Dugas - S. Feigelstock, Self-free modules and E-rings. Comm. Algebra 31 (2003), no. 3, pp. 1387-1402.

[6] M. DuGAS, Localizations of torsion-free abelian groups. J. Algebra 278 (2004), pp. 411-429.

[7] M. Dugas, Localizations of torsion-free abelian groups II. J. Algebra 284 (2005), no. 2, pp. 811-823.

[8] A.A. Fomin, Tensor products of torsion-free abelian groups, Siberian Math. J. 16 (1975), no. 5, pp. 820-827.

[9] R. GöBel - B. Goldsmith - O. Kolman, On modules which are self slender. Houston J. Math. 35 (2009), no. 3, pp. 725-736.

[10] R. GöBel - D. HeRden - S. ShelaH, Absolute E-rings. Adv. Math. 226 (2011), no.1, pp. 235-253.

[11] R. GöBEL - J. TRLifaJ, Approximations and endomorphism algebras of modules. Second extended edition. de Gruyter Expositions in Mathematics, 41. Walter de Gruyter GmbH \& Co. KG, Berlin, 2012.

[12] T.A. Hungerford, Algebra, Graduate Texts in Mathematics, Springer-Verlag New York Inc. 1974.

[13] R.E. Johnson - E.T. Wong, Quasi-injective modules and irreducible rings. J. London Math. Soc. 36 (1961), pp. 260-268.

Manoscritto pervenuto in redazione il 29 Giugno 2013. 